\title{
A influência da desintegração do Radônio transportado pela evapotranspiração das plantas na formação de aerossóis atmosféricos em ambiente florestal
}

\author{
Motoki, K. ${ }^{\text {a }}$, Campos, T.F.C. ${ }^{\text {b }}$ Pastura V.F.S. ${ }^{c}$, Sichel S.E. ${ }^{a}$, Fonseca E. M. ${ }^{a}$ \\ ${ }^{a}$ Instituto de Geociências da Universidade Federal Fluminense, Gragoatá, Niterói - RJ, 24210-346. ${ }^{b}$ Departamento de \\ Geologia da Universidade Federal do Rio Grande do Norte, Campus Central da UFRN, Lagoa Nova Natal RN 59064 - \\ 741; 'Instituto de Energia Nuclear da Comissão Nacional de Energia Nuclear, Ilha do Fundão, \\ Rio de Janeiro - RJ, 21941-614
}

e-mail:kmotoki.mag@gmail.com; thomascampos@geologia.ufrn.br; vpastura@ien.gov.br; susannasichel@id.uff.br; oceano25@hotmail.com

\section{RESUMO}

Entre as diferentes variáveis que provocam impactos no clima, devemos contar com a variação dos aerossóis atmosféricos, nesse contexto e excluindo-se a atividade humana, o seu aparecimento está intimamente correlacionado com a presença de íons na atmosfera. Essas moléculas ionizadas são formadas tanto pelas ações dos raios cósmicos espaciais, como pelas radiações Gama e Alfa terrestres, que irão constituir os aglomerados iônicos que, por sua vez, influenciam na quantidade de aerossóis atmosféricos de uma dada região, principalmente em áreas florestais. Esse fato será devido ao transporte de gás Radônio dissolvido nas águas subterrâneas para o ar pela evapotranspiração das plantas, uma vez que a alta densidade do gás Radônio (10 $\mathrm{kg} / \mathrm{m}^{3}$ ) liberado diretamente de solos e rochas tem pouco poder de penetração na atmosfera. Nesse artigo apresenta-se um estudo sobre a correlação de partículas iônicas em uma atmosfera de floresta insular (ilha da Trindade) e de mata atlântica dunar (parque da Dunas, Natal RN) com a variação do gás Radônio.

Palavras-chave: Radônio, Aerossóis atmosféricos, Clima florestal. 


\begin{abstract}
Among the different variables that cause impacts on the climate, we must rely on the variation of atmospheric aerosols, in this context and excluding human activity, its appearance is closely correlated with the presence of ions in the Atmosphere. These ionized molecules are formed both by the actions of cosmic space rays and by the terrestrial Gamma and Alpha radiations that will constitute the ionic agglomerates which, in turn, influence the amount of atmospheric aerosols of a given region, especially in forest areas. This will be due to the transport of Radon gas dissolved in the groundwater to the air by evapotranspiration of the plants, since the high density of the Radon gas $\left(10 \mathrm{~kg} / \mathrm{m}^{3}\right)$ released directly from soils and rocks has little penetration power in the Atmosphere. In this paper we present a study on the correlation of ionic particles in an island forest atmosphere (Trindade Island) and Atlantic dune forest (Dunas Park, Natal RN) with the variation of Radon gas.
\end{abstract}

Keywords: Radon, Atmospheric aerosols, Forest climate. 


\section{INTRODUÇÃO}

A variação dos aerossóis atmosféricos provoca grande impacto no clima de uma da região. Pesquisas internacionais identificaram a existência de uma correlação intrínseca entre esses aerossóis atmosféricos e à presença de íons na atmosfera. Esses aglomerados iônicos se formam tanto pela radiação cósmica, como pelas radiações terrestres Gama e Alfa. Nesse contexto, chamase atenção para a radiação ionizante proveniente do Radônio 222 e seus descendentes em áreas florestal, pois devido ao transporte de gás Radônio dissolvido nas águas subterrâneas para o dossel florestal, através da evapotranspiração vegetal, uma vez que a alta densidade do gás radônio (10 $\mathrm{kg} / \mathrm{m}^{3}$ ) exalado dos solos e rochas tem pouco poder de penetração na atmosfera baixa.

Os aglomerados de íons na Atmosfera são formados pela ação da radiação cósmica, da radiação Gama da superfície terrestre e da radiação Alfa do Radônio existentes no Ar. Admite-se que a concentração de aglomerados de íons esteja diretamente relacionada com o nível de radônio na Atmosfera, como foi demonstrado por [1] nos seus estudos sobre a exalação de radônio nas cavernas da república Checa e por [2] nas florestas australianas. Esses autores constataram uma forte correlação entre os níveis de radônio e os íons positivos $\left(\mathrm{R}^{2}=0,89\right)$ e íons negativos $\left(\mathrm{R}^{2}=\right.$ 0,96) na atmosfera. Este fato também foi identificado por [3] que demonstraram que as Medianas desses aglomerados em zonas florestais $\left(725 \mathrm{~cm}^{3}\right)$ eram significativamente superiores às de áreas abertas $\left(269 \mathrm{~cm}^{3}\right)$.

O semiárido brasileiro compreende uma área de quase um milhão de $\mathrm{km}^{2}$, abrange à maior parte dos estados nordestinos. Além da ocorrência da seca, essa região é caracterizada pela má distribuição das águas e chuvas, elevada evapotranspiração, alta salinidade. Por sua vez, o clima da ilha Trindade é do tipo oceânico tropical, com temperatura média anual de $25^{\circ} \mathrm{C}$, sendo o mês de fevereiro o mais quente $\left(30^{\circ} \mathrm{C}\right)$ e o de agosto o mais frio, com temperatura em torno de $17^{\circ} \mathrm{C}$. De abril a outubro a ilha sofre invasão periódica de frentes frias provindas do sul. A precipitação média anual é de $923 \mathrm{~mm}$, mas muito variável, o seu sistema de drenagem é pouco expressivo, com de baixa vazão [4].

Partindo desses pressupostos, se faz necessário a realização de esforços de investigação, de maneira a captar e descrever as tendências de distribuição de radônio na atmosfera baixa das áreas 
de florestas naturais e seu entorno degradado, criando-se um quadro de referência teórico-prático de maneira a sistematizar e compreender as transformações em curso, e aplicá-lo aos processos de mudanças climáticas.

Nesse trabalho apresentam-se os resultados sobre a correlação do teor de Radônio e com os aglomerados iônicos (+ e -) nos dosséis da floresta de Samambaia (Cyathea Sm.) da Ilha Trindade (Sudeste do Brasil) e da Mata Atlântica dunar do município de Parnamirim, estado do Rio Grande do Norte (Nordeste do Brasil).

\section{MATERIAIS E MÉTODOS}

Para as medições ativas e passivas do gás radônio na atmosfera foi usado um emanômetro AlphaGuard® PRO (GENITRON INC). As medições dos aglomerados de íons atmosféricos foram realizadas através de um contador de íons (Air Ions Counter da AlphaLab® INC). A radiação Gama superficial foi medida através de um espectrômetro de radiação Gama modelo RS-230® (RADIATION SOLUTION INC.). Por sua vez, as condições atmosféricas locais foram medidas através de estação meteorológica portável modelo VANTAGE PRO 2 (DAVIS INC.). Os equipamentos necessários foram disponibilizados pelo Laboratório de Radioatividade Natural do Departamento de Geologia da Universidade Federal do Rio Grande do Norte.

Os protocolos de medição seguidos na montagem da torre de medição de $5 \mathrm{~m}$ de altura foram os seguintes:

a) A medições ativas de radônio foram realizadas a cerca de $1 \mathrm{~m}$ do solo;

b) As medições de íons atmosféricos (+ e -) foram realizadas a 1 e $5 \mathrm{~m}$ do solo ;

c) As condições meteorológicas foram medidas através de uma estação meteorológica fixada a $1 \mathrm{~m}$ e a outra a $5 \mathrm{~m}$ do solo.

Considerando o tempo de meia-vida do Radônio ( $\approx 3,8$ dias), a duração do período de coleta de dados escolhidos foi de 5 dias. Como o clima no interior do nordeste brasileiro é bem peculiar, não existindo estações do ano marcantes, quando muito resume-se a duas estações: a "época de chuva" e 
a "época de seca", seca essa que pode durar anos, devido então a essa peculiaridade, optou-se realizar as medições no "período seco" e em caso de chuva esporádica só realizar medições após três dias de estiagem. Contudo, também dada a peculiaridade da variação meteorológica da ilha Trindade, as medições de Radônio só ocorreram nos períodos da semana que não chovesse durante três dias.

\subsection{Aspectos geológicos e biológicos}

O estado do Rio Grande do norte é conhecido pelas suas regiões granítico-pegmatítica e dunar enriquecidas em minerais que contem Urânio e Tório. Grande parte dessas regiões estão cobertas por floresta atlântica dunar e por caatinga. A floresta atlântica é um bioma terrestre e compreende uma região que se estende ao longo da costa atlântica brasileira, desde o Rio Grande do Norte, no Nordeste, até o Rio Grande do Sul, no sul do país (Fig. 1A, B). Por sua vez, a ilha Trindade possui um substrato vulcânico de caráter sódico-alcalino e de piroclastos diversos. A diversidade de solos está profundamente relacionada com as variações do substracto vulcânico e sua posição altimétrica (Fig. 1C, D).

De maneira geral, os solos na ilha Trindade apresentam baixa fertilidade natural e grau de intemperismo acentuado. Esse fato deve-se ao primeiro desastre ecológico em solo brasileiro, pois segundo narrativas históricas, o comandante do navio inglês $H$. M. S. Paramore, que trazia o famoso astrônomo Edmund Halley, soltou os primeiros casais de cabras, porcos e carneiros na ilha, bem como pela tentativa portuguesa de colonizar a ilha da Trindade.

O processo de colonização da ilha da Trindade foi historicamente abandonado, devido a irregularidade do substracto vulcânico, que não permitia o seu aplainamento para a agricultura e os animais que foram abandonados e se proliferaram às custas da vegetação nativa, devastando as florestas que cobriam pelo menos $85 \%$ da Ilha da Trindade. A maior parte da degradação se deu entre a visita de Halley e a primeira metade do século XIX [5].

Atualmente, a ilha da Trindade é coberta por gramíneas (60\%) em áreas abertas e por vegetação arbóreas (5\%), nomeadamente por samambaias gigantes (Cyathea copelandii Kuhn \& Luerss) e muito raramente a Colubrina glandulosa Perk, que sobreviveram a devastação caprina, nas altas altitudes (400 a 550 m) devido a sua casca não ser comestível por esses animais (Fig. 1C, D). 


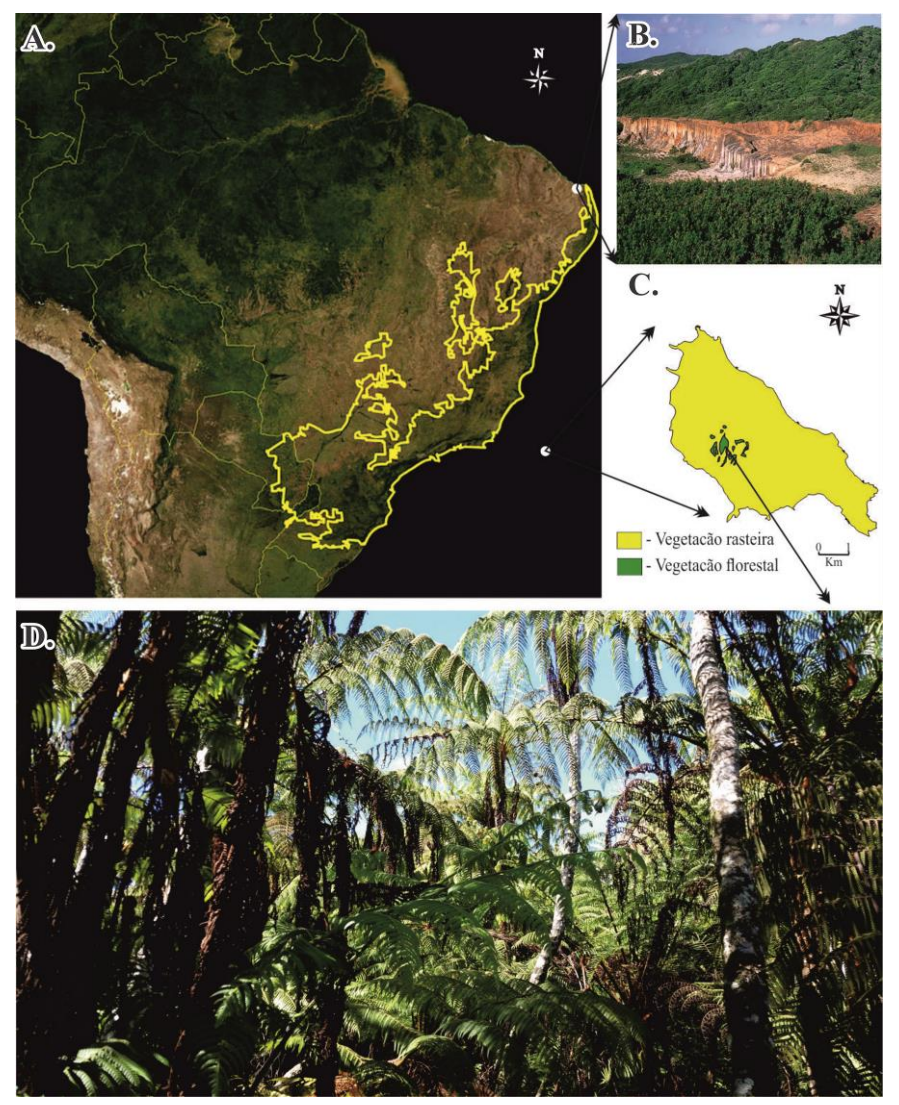

Figura 1: Localização das áreas pesquisadas: A) Demarcação da área da Mata Atlântica no Brasil, em verde são as matas existentes (apud WWF) B) Parte da área Mata Atlântica Dunar, no Rio Grande do Norte (Natal); C) Área de florestal da ilha da Trindade: em amarelo a cobertura de liquens, gramíneas e herbáceas, em verde a floresta arbóreas, com predomínio de Samambaias

(Cyathea Sm.) e muito mais raramente Colubrina granulosa; D) Parte da área da floresta de samambaias gigantes da ilha Trindade. Fonte: Figura 1A: Imagem de satelite da NASA

\section{RESULTADOS E DISCUSSÃO}

As medições de íons atmosféricos e de Radônio foram realizadas, tanto no interior, como fora das florestas e no sentido contrário ao vento (Tabela 1 e 2, Fig. 2). Apesar das medições terem sido realizadas ao longo de 5 dias, os dados sobre os íons e Radônio aqui analisados estão restritos às horas de luz do dia, em condições climáticas favoráveis, com a temperatura do ar entre 20 e $30^{\circ} \mathrm{C}$, de modo a se evitar as conhecidas variações diurnas acentuadas nas concentrações do Radônio e dos íons atmosféricos. Por isso estão apresentadas a média geométrica das medições. 
Tabela 1: Média geométrica do teor total de íons atmosféricos em diferentes regiões floretais no Brasil

\begin{tabular}{|c|c|c|c|c|c|c|c|c|c|c|}
\hline \multirow[b]{3}{*}{ Hora } & \multicolumn{10}{|c|}{ Íons totais } \\
\hline & \multicolumn{4}{|c|}{ Illha da Trindade } & \multicolumn{4}{|c|}{ Parque das Dunas } & \multirow{2}{*}{$\begin{array}{c}\text { Amazônia } \\
\text { MG* } \\
\text { Dentro da } \\
\text { floresta }\end{array}$} & \multirow{2}{*}{$\begin{array}{c}\text { Índia } \\
\text { MG* Fora } \\
\text { da } \\
\text { floresta }\end{array}$} \\
\hline & $\begin{array}{c}\text { MG* }^{*} \\
\text { Dentro da } \\
\text { floresta }\end{array}$ & DP** & $\begin{array}{l}\text { MG* }^{*} \\
\text { Fora da } \\
\text { floresta }\end{array}$ & DP** & $\begin{array}{c}\text { MG* }^{*} \\
\text { Dentro da } \\
\text { floresta }\end{array}$ & DP & $\begin{array}{c}\text { MG* } \\
\text { Fora da } \\
\text { floresta }\end{array}$ & $\mathbf{D P}^{* * *}$ & & \\
\hline $07: 41$ & 184 & 0,3 & 34 & 0,2 & 170 & 0,5 & 65 & 0,6 & 700 & 388 \\
\hline 08:41 & 209 & 0,3 & 49 & 0,2 & 130 & 0,5 & 75 & 0,6 & 685 & 333 \\
\hline 09:41 & 222 & 0,4 & 52 & 0,3 & 162 & 0,6 & 78 & 0,7 & 640 & 315 \\
\hline $10: 41$ & 234 & 0,2 & 74 & 0,1 & 135 & 0,4 & 80 & 0,5 & 525 & 197 \\
\hline $11: 41$ & 247 & 0,5 & 77 & 0,4 & 170 & 0,7 & 95 & 0,8 & 455 & 168 \\
\hline $12: 41$ & 269 & 0,3 & 89 & 0,2 & 180 & 0,5 & 96 & 0,6 & 415 & 145 \\
\hline $13: 41$ & 247 & 0,4 & 107 & 0,3 & 154 & 0,5 & 89 & 0,7 & 373 & 134 \\
\hline $14: 41$ & 264 & 0,4 & 114 & 0,3 & 130 & 0,5 & 89 & 0,6 & 364 & 125 \\
\hline $15: 41$ & 254 & 0,4 & 134 & 0,3 & 100 & 0,6 & 70 & 0,7 & 368 & 147 \\
\hline $16: 41$ & 307 & 0,4 & 147 & 0,3 & 160 & 0,6 & 100 & 0,7 & 389 & 138 \\
\hline $17: 41$ & 320 & 0,3 & 162 & 0,2 & 205 & 0,5 & 110 & 0,6 & 446 & 149 \\
\hline $18: 41$ & 342 & 0,4 & 172 & 0,3 & 210 & 0,5 & 115 & 0,7 & 460 & 163 \\
\hline $19: 41$ & 490 & 0,4 & 290 & 0,3 & 165 & 0,6 & 118 & 0,7 & 450 & 153 \\
\hline $20: 41$ & 933 & 0,2 & 333 & 0,1 & 180 & 0,4 & 121 & 0,5 & 489 & 192 \\
\hline $21: 41$ & 875 & 0,4 & 375 & 0,3 & 182 & 0,6 & 134 & 0,7 & 570 & 273 \\
\hline $22: 41$ & 964 & 0,4 & 414 & 0,3 & 205 & 0,5 & 138 & 0,7 & 550 & 298 \\
\hline
\end{tabular}

*MG: Média geométrica; **DP: Desvio padrão

Tabela 2: Média geométrica do teor de Radônio na atmosfera de diferentes regiões floretais no Brasil

\begin{tabular}{|c|c|c|c|c|c|c|c|c|c|c|}
\hline \multirow[b]{3}{*}{ Hora } & \multicolumn{10}{|c|}{ Radônio $\left(\mathbf{k B q} / \mathbf{m}^{3}\right)$} \\
\hline & \multicolumn{4}{|c|}{ Illha Trindade } & \multicolumn{4}{|c|}{ Parque das Dunas } & \multirow{2}{*}{$\begin{array}{c}\text { Amazônia } \\
\text { MG* } \\
\text { Dentro da } \\
\text { floresta }\end{array}$} & \multirow{2}{*}{$\begin{array}{c}\text { Índia } \\
\text { MG* Fora } \\
\text { da } \\
\text { floresta }\end{array}$} \\
\hline & $\begin{array}{c}\text { MG* }^{*} \\
\text { Dentro da } \\
\text { floresta }\end{array}$ & $\mathbf{D P} * *$ & $\begin{array}{c}\text { MG* } \\
\text { Fora da } \\
\text { floresta }\end{array}$ & DP** & $\begin{array}{c}\text { MG* }^{*} \\
\text { Dentro da } \\
\text { floresta }\end{array}$ & DP** & $\begin{array}{c}\text { MG* }^{*} \\
\text { Fora da } \\
\text { floresta }\end{array}$ & $\mathbf{D P} * *$ & & \\
\hline $07: 41$ & 20 & 3 & 12 & 1,7 & 57 & 2,5 & 42 & 2 & 22 & 17 \\
\hline 08:41 & 25 & 2,2 & 13 & 0,9 & 75 & 2,7 & 53 & 2,2 & 24,9 & 14 \\
\hline 09:41 & 27 & 2,8 & 16 & 1,5 & 78 & 2,6 & 65 & 2,1 & 25 & 13 \\
\hline 10:41 & 26 & 2,9 & 15 & 1,6 & 81 & 2,7 & 71 & 2,2 & 22 & 10 \\
\hline 11:41 & 23 & 2,7 & 14 & 1,4 & 82 & 2,7 & 73 & 2,2 & 16 & 8 \\
\hline $12: 41$ & 22 & 2,8 & 13 & 1,5 & 84 & 2,5 & 71 & 2 & 14 & 6 \\
\hline $13: 41$ & 24 & 3 & 14 & 1,7 & 81 & 2,4 & 69 & 1,9 & 15 & 7 \\
\hline 14:41 & 27 & 3 & 16 & 1,7 & 80 & 2,7 & 66 & 2,2 & 18 & 8 \\
\hline $15: 41$ & 29 & 3,1 & 18 & 1,8 & 76 & 2,8 & 66 & 2,3 & 19 & 12 \\
\hline $16: 41$ & 35 & 2,5 & 22 & 1,2 & 78 & 2,7 & 69 & 2,2 & 20 & 14 \\
\hline $17: 41$ & 37 & 3 & 24 & 1,7 & 81 & 2,4 & 70 & 1,9 & 21 & 14 \\
\hline 18:41 & 41 & 3 & 25 & 1,7 & 82 & 2,8 & 71 & 2,3 & 21 & 15 \\
\hline 19:41 & 48 & 2,5 & 28 & 1,2 & 81 & 2,4 & 71 & 1,9 & 18 & 16 \\
\hline 20:41 & 50 & 2,6 & 33 & 1,3 & 79 & 2,7 & 67 & 2,2 & 17 & 12 \\
\hline $21: 41$ & 58 & 2,7 & 38 & 1,4 & 75 & 2,6 & 64 & 2,1 & 16 & 11 \\
\hline $22: 41$ & 60 & 2,9 & 47 & 1,6 & 69 & 2,6 & 63 & 2,1 & 15 & 10 \\
\hline
\end{tabular}

*MG: Média geométrica; **DP: Desvio padrão 
O radônio é um gás mais pesado que o ar e em condições atmosféricas estáveis, como o que ocorre durante a noite e no início da manhã, tende a acumular-se perto do solo. A turbulência devido ao aquecimento solar durante o dia causar uma grande difusão turbulenta (difusão eddy) na atmosfera, que pode diluir o radônio que emana dos solos. Além disso, nas áreas estudadas, existem boas evidências para sugerir que a concentração de radônio na atmosfera segue uma tendência diurna, semelhante à concentração de aglomerados de íons, com um máximo no início da manhã e um mínimo na parte da tarde.

Na Figura 2 apresenta-se os gráficos com as variações diurnas dos teores de Radônio e íons provenientes de observações nos diferentes tipos de florestas (dentro e fora delas): Floresta Atlântica do Parque das Dunas (Natal-RN) no Nordeste do Brasil, floresta de samambaias gigantes da Ilha da Trindade no Sudeste do Brasil. A título de comparação, nesses gráficos também projetase os dados sobre florestas de Mysore (Índia) [6] e da Amazônia brasileira [7]. Todas as curvas apresentam o tradicional pico de Radônio pela manhã (Fig. 2), cabe salientar que devido a escala vertical do gráfico do Radônio ser vasta, alguns picos matinais foram atenuados. No entanto, nesses gráficos pode-se ver que há um segundo pico que aparece à tarde (Fig. 2). Outros estudos também relataram esse mesmo fenômeno, como pode ser visto nos dados de [2], [6] e [7].

Os resultados obtidos sobre as variações do Radônio e de Íons Total são similares aos existentes na literatura, isto é, seguem a já conhecida variação diurna do gás radônio, similarmente a variação dos íons, onde há um crescimento nas primeiras horas da manhã, uma diminuição ao meio do dia e um aumento para o final da tarde. Contudo, existem diferenças que se associa ao substrato geológico, o tipo de solo, o clima e ao tipo de vegetação florestal (Fig. 2), pois os valores de Radônio e de íons nas áreas estudadas são mais elevados do que os divulgados na literatura. 
(A)

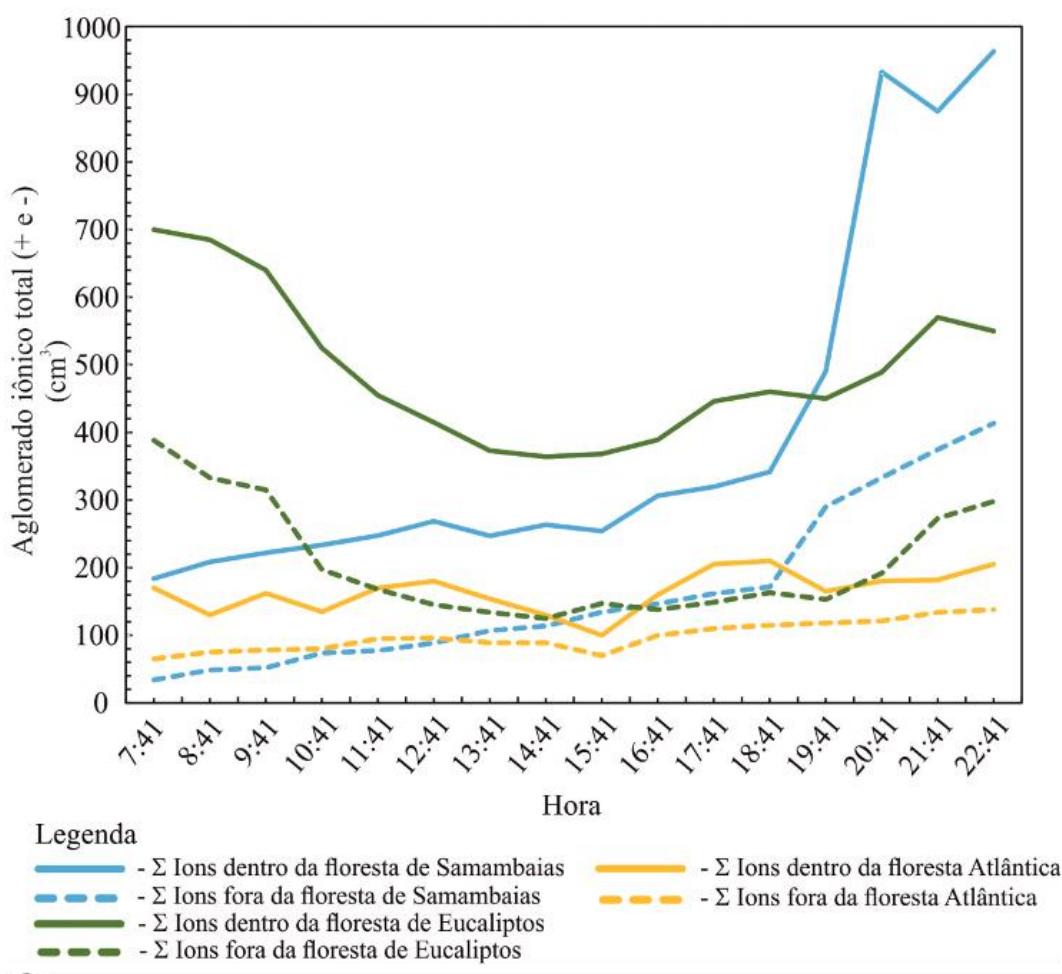

(B)

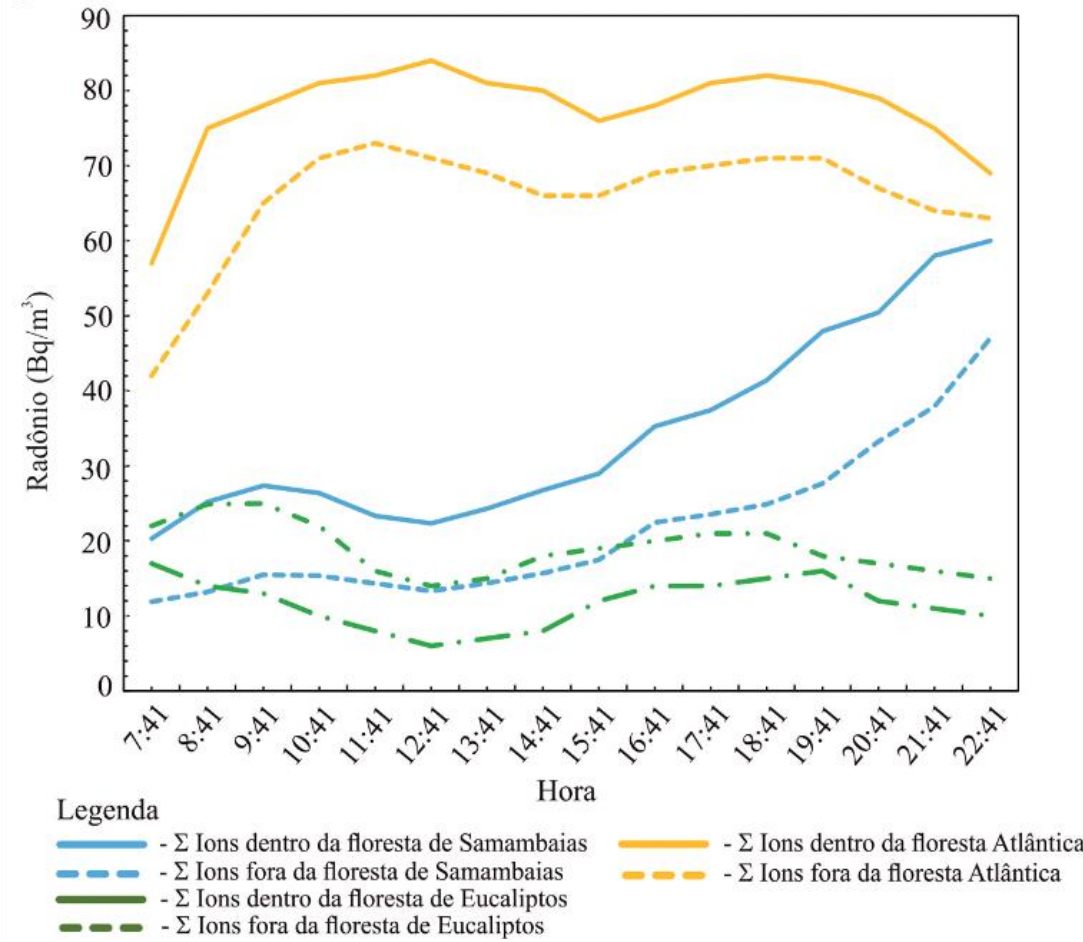

Figura 2 : A) Variação dos íonstotal (+ $e$-) ao longo do dia, em diferentes tipos de florestas; B) Variação do gás Radônio ao longo do dia, em diferentes tipos de florestas. 


\section{CONCLUSÕES}

A temperatura e turbulência do Ar geralmente possuem picos durante o meio da tarde e, consequentemente, tanto a concentração do radônio, como à dos íons não são esperadas aumentarem nesse período. Assim, conclui-se que esses picos ao meio da tarde são relacionados à liberação de Radônio para a atmosfera pela evapotranspiração das plantas que, geralmente, ocorrem durante a tarde, conclusão essa que corrobora com à de [2]. Contudo, os resultados deste trabalho quando comparado com os das outras regiões apresentam os picos máximos e mínimos do radônio e dos aglomerados iônicos com um certo atraso ao longo do dia. Fato esse que é devido tanto à pouca variação da temperatura do ar ao longo do dia, como aos teores de Radônio serem mais altos em relação as outras regiões. O fato do Radônio ser mais alto é devido a influência geológica. Visto que os solos e do Parque florestal das Dunas - Natal são constituído por areias eólicas enriquecidas em minerais pesados portadores de Urânio e Tório (Monazita, Alanita, Epidoto e Thorianita) e a floresta de samambaias da ilha da Trindade se encontrar sobre rochas vulcânicas fonolíticas, rochas essas que possuem Urânio disperso em sua matriz.

\section{AGRADECIMENTOS}

Esta pesquisa foi financiada pelo Conselho Nacional de Pesquisa e Desenvolvimento Tecnológico do Brasil (CNPq) e recebeu apoio laboratorial do Instituto de Engenharia Nuclear e do Laboratório de Poços de Caldas do Comitê Nacional de Energia Nuclear (IEN/LAPOC/CNEN) e apoio logístico do Programa Arquipélago e Ilhas Oceânicas da Secretaria Interministerial de Recursos do mar (SECIRM) e da Marinha do Brasil.

\section{REFERÊNCIAS}

[1] Sas, D.; Sladek, P.; Navatril, O. Radon and air ion balance. Czech. J. Phys. 2006, 56, 141-148.

[2] Jayaratne, E.R. Ling X., Morawska L. 2011 The Role of Vegetation in Enhancing Radon concentration and Ion Production in the Atmosphere. Environ. Sci. Technol., 2011, 45 (15), pp $6350-6355$ 
[3] Ling, X.; Jayaratne, E. R.; Morawska, L. 2010. Air ion concentrations in various urban outdoor environments. Amospheric Environment, 44, 2186-2193.

[4] Clemente, E. P.; Schaefffer, C. E.; Oliveira, F. S.; Albuquerque Filho, M. R.; Alves, R. J.; Firme SÁ, M. M.; Melo, V. F.; Corrêa, G. R. Topossequência de solos na Ilha da Trindade, Atlântico Sul. Rev. Bras. Ciênc. Solo, v. 33 n. 5, 2009.

[5] Faria, A.L.A (2012): As briófitas da Ilha da Trindade, Atlântico Sul, Brasil. Dissertação (Mestrado, Universidade de Brasília, Instituto de Biologia, Departamento de Botânica, Programa de Pós-Graduação em Botânica, 2012, 63 pp)

[6] Martens, C.S., Shay, T.J., Mendlovitz, H.P., Matross, D.M., Saleska, S.R., Wofsy, S.C., Woodward, W.S., Menton, M.C., De Moura, J.M.S., Crill, P.M., De Moraes, O.L.L., Lima, R.L. Radon fluxes in tropical forest ecosystems of Brazilian Amazonia: nighttime CO2 net ecosystem exchange derived from radon and eddy covariance methods. Global Change Biol. 10 (5), 618629, 2004.

[7] Chandrashekara, M. S.; Sannappa, J.; Paramesh, L. 2006. Studies on atmospheric electrical conductivity related to radon and its progeny concentrations in the lower atmosphere at Mysore. Atmospheric Environment, 40, 87-95. 